\section{In Memoriam \\ Medizinalrat Dr. med. Joachim Bernhard}

Medizinalrat Dr. med. Joachim Bernhard ( $\bullet$ Abb. 1), ehemaliger Leiter der Unfallchirurgischen Abteilung an der Chirurgischen Klinik der Medizinischen Akademie „Carl Gustav Carus“ Dresden, verstarb im 87. Lebensjahr am 15. Mai 2012 in Dresden. In dieser Stadt wurde er am 1. Januar 1925 als Sohn des Verwaltungsrats Oskar Bernhard und dessen Ehefrau Ella geboren und verbrachte hier eine glückliche Kindheit, liebevoll von den Eltern betreut und behütet. Joachim Bernhard besuchte von 1931 bis 1935 die Volksschule und anschließend bis 1943 das Realgymnasium Dresden Blasewitz mit erfolgreichem Abiturabschluss. Unmittelbar danach folgte für 2 Monate der Arbeitsdiensteinsatz und Juli 1943 die Einberufung zur Wehrmacht. Nach der Grundausbildung war Joachim Bernard als Funker an der Westfront eingesetzt und geriet am Kriegsende in amerikanische Gefangenschaft, aus der er am 20. Dezember 1945 entlassen wurde. Diese 2 Jahre Kriegsdienst waren für den 18-jährigen Abiturienten Erlebnisjahre, an die er sich ungern erinnerte, da der verlustreiche Rückzug unter den anhaltenden Bombardements und Zerstörungen recht schnell die Sinnlosigkeit des Krieges erkennen ließ.

Schon im Januar 1946 nahm Joachim Bernhard eine Tätigkeit als Krankenpfleger in dem teils schwer zerstörten Krankenhaus Dresden-Johannstadt auf und bemühte sich um einen Studienplatz für Medizin. Die Immatrikulation erfolgte im Oktober 1946 an der Medizinischen Fakultät der Universität Rostock, d.h. Bernhard begann sein Medizinstudium im ersten Semester nach der Wiedereröffnung der Universität. Das Medizinstudium in dieser unmittelbaren Nachkriegszeit war mit alltäglichen Schwierigkeiten verbunden, die jedoch den Studenten Bernhard von seinem unabänderlichen Wunsch, Arzt zu werden, nicht beeinflussten, sodass der Studiumverlauf streng eingehalten wurde und das Staatsexamen im Mai 1952 und im gleichen
Monat auch die Promotionsleistungen zum Dr. med. erfolgreich abgeschlossen werden konnten.

Nach dem Medizinstudium kehrte Bernhard sofort nach Dresden zurück und absolvierte vom 15. Juni 1952 an seine 2jährige Pflichtassistenzzeit im Krankenhaus Dresden-Johannstadt; hier fanden die Fächer Chirurgie und Orthopädie sein großes Interesse und nach Erhalt der Vollapprobation begann er 1954 mit der Weiterbildung zum Facharzt für Orthopädie. Sein Lehrer war Prof. Dr. med. J. Büschelberger, der noch weitgehend die konservative Orthopädie vertrat und den Assistenten eine tradierte Ausbildung und konservative Erfahrungswerte vermittelte. 1958 wurde Dr. med. Joachim Bernhard zum Facharzt für Orthopädie ernannt, übernahm 1959 die Leitung der orthopädischen Abteilung am Krankenhaus Radebeul und gleichzeitig die Funktion des Kreisorthopäden Dresden Land. Diese konservative Orthopädie wie auch die von Amts wegen auszuführende bürokratische Funktion befriedigten keineswegs die von Dr. Bernhard so gewünschten operativen Ambitionen und schon nach 4 Monaten entschloss er sich, in die Carl-Gustav-Carus-Akademie zurückzukehren, aber jetzt in die Chirurgie zu Prof. Hans Bernhard Sprung, den er sehr verehrte. Im Januar 1960 begann er mit der chirurgischen Weiterbildung und erhielt schon am 1. Juli 1961 die Facharztanerkennung für Chirurgie; er war vorrangig in der Behandlung unfallchirurgischer Patienten tätig. 1962 wurde Dr. Joachim Bernhard zum Oberarzt an der Chirurgischen Klinik der Medizinischen Akademie ernannt und mit der Wahrnehmung des Fachbereichs Unfallchirurgie betraut. Nach dem Tod von Prof. Dr. Sprung wurde Prof. Dr. Richard Kirsch 1963 auf den vakanten Lehrstuhl für Chirurgie berufen. Oberarzt Dr. Joachim Bernhard wurde nunmehr Leiter der Traumatologischen Abteilung und übernahm auch die Vorlesungen in Traumatologie in der Hauptvorlesung Chirurgie.

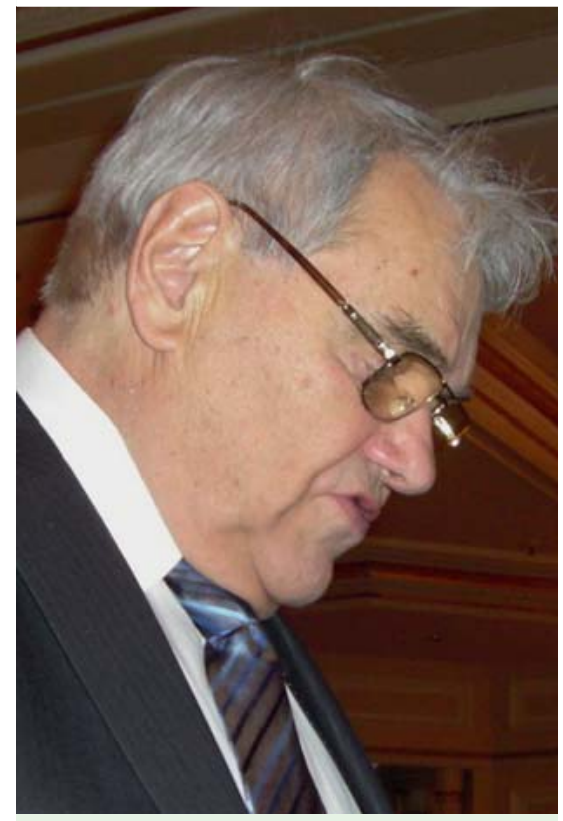

Abb. 1 MR Dr. Joachim Bernhard.

Als Traumatologe des Hauses konnte Dr. Joachim Bernhard auch eigene Vorstellungen und Akzente setzen. In den 60erJahren rückte die operative Versorgung auch von älteren Patienten immer mehr in den Vordergrund, besonders die Marknagelung nach Küntscher zur Versorgung bei Frakturen der Röhrenknochen und die intramedulläre Fixation von Schenkelhalsbrüchen. Besondere Aufmerksamkeit erlangte die Verwendung des Rundnagels nach Lezius-Herzer bei trochantären Frakturen, sie wurde von Dr. Bernhard als Standardmethode eingeführt und methodisch adaptiert, sodass ausgezeichnete Ergebnisse erreicht werden konnten, die in mehreren Publikationen, auch als Spätergebnisse, Anerkennung fanden. Wissenschaftlich bearbeitet wurden auch die Meniskusläsionen, wo eine Verbesserung in der damaligen Diagnostik mithilfe von arthrografischen Untersuchungen des Kniegelenks erreicht wurde. Aus dem traumatologischen Arbeitsgebiet erschienen von Dr. Bernhard über 30 Publikationen und über 55 Vorträge wurden gehalten.

Nach meiner Berufung 1972 auf den Lehrstuhl für Chirurgie und Ernennung zum Klinikdirektor an der Medizinischen Akademie Carl Gustav Carus lernte ich Dr. Joachim Bernhard, den Leiter der Abteilung für Traumatologie, kennen und konnte mich schon nach wenigen Monaten von der verantwortungsvollen Indikationsstellung, der exzellenten operativen Technik und von dem menschlichen 
und ärztlichen Tun überzeugen. Seine stete Einsatzfreudigkeit und ärztliche $\mathrm{Zu}$ wendung den Schwerverletzten und älteren Patienten gegenüber zeichneten ihn aus. Die Traumatologische Abteilung wurde von Dr. Bernhard als selbstständige Fachabteilung bestens geleitet und weiterentwickelt und so die Stabilität in der Versorgung Unfallverletzter für über 25 Jahre an der Medizinischen Akademie gesichert. Diese seine Leistungen fanden Anerkennung durch Ehrungen und Auszeichnungen, so durch die Verleihung der Medaille für ausgezeichnete Leistungen 1966, die Ehrenurkunde und Medaille in Silber für treue Dienste zum 20-jährigen Dienstjubiläum 1973 und in Anerkennung ständiger Einsatzbereitschaft zum 30. Dienstjubiläum die Medaille in Gold 1983. Mit der Verleihung des Titels „Medizinalrat“ 1980 wurde die hervorragende Leitungstätigkeit beim Aufbau der Traumatologischen Abteilung gewürdigt.
Nach dem altersbedingten Ausscheiden von Medizinalrat Joachim Bernhard 1990 aus der Medizinischen Akademie war die erreichte Freizeit und Freiheit teilweise durch krankheitsbedingte Störungen eingeschränkt. Auch in dieser Situation stand ihm seine über alles liebende Frau Renate zur Seite. Die Eheschließung erfolgte 1955 nach der Facharzternennung für Orthopädie, alle seine weiteren Schritte vollzog er in Übereinstimmung und Zuneigung. Und so verflossen auch diese schönen Jahre in Freundschaft mit Weggefährten und Treffen mit Kollegen. Das eigene Haus, das das Ehepaar Dr. Bernhard 1968 in der schönen Lage Dresdens erbaute, war das Refugium der Zweisamkeit. Aus scheinbar voller Gesundheit schied Medizinalrat Dr. Joachim Bernhard in wenigen Stunden am 15. Mai 2012 aus dem Leben und hinterließ seine so liebenswürdig geschätzte fassungslose Ehefrau Renate in Trauer.
Alle, die Dr. Joachim Bernhard kannten, werden ihn in guter Erinnerung behalten als freundlichen, entgegenkommenden Kollegen und Mitmenschen, als hingebungsvollen Arzt und treuen Freund.

\section{Interessenkonflikt: Nein}

H. Wolff

Berlin, Deutschland

\section{Bibliografie}

Dol http://dx.doi.org/10.1055/s-1328249

Zentralbl Chir 2013; 138: 104-105

(c) Georg Thieme Verlag KG Stuttgart · New York . ISSN 0044-409X

Korrespondenzadresse

Prof. Dr. Helmut Wolff

Rapsweg 26

12683 Berlin

Deutschland

Tel.: 030/5413833

helmutwolff@kabelmail.de 